\title{
Evolution of microcontroller-based remote monitoring system applications
}

\author{
Wael A. Salah' ${ }^{1}$, Basem Abu Zneid ${ }^{2}$ \\ ${ }^{1}$ Department of Electrical Engineering, College of Engineering and Technology, \\ Palestine Technical University-Kadoorie, Palestine \\ ${ }^{2}$ Faculty of Electrical and Computer Engineering, University of Business and Technology CEiT, Saudi Arabia
}

\begin{abstract}
Article Info
Article history:

Received Jun 2, 2018

Revised Jan 28, 2019

Accepted Mar 6, 2019

\section{Keywords:}

Microcontroller

Remote monitoring

Smart application

Wireless/wired
\end{abstract}

\begin{abstract}
This study reviews the evolution of smart applications of microcontrollerbased wireless/wired remote monitoring systems. Rapid developments in science and technology offer the advantages of using integrated embedded chips, microprocessors, and microcontrollers. The use of microcontrollers in industrial processes, such as automobiles, aeronautics, space, robotics, electronics, defense applications, mobile communications, rail transport, and medical applications, is rapidly increasing. This study aims to review the progress of microcomputers in smart remote monitoring and controlling applications for the control and management of different systems using wireless/wired techniques.
\end{abstract}

Copyright $@ 2019$ Institute of Advanced Engineering and Science. All rights reserved.

Corresponding Author:

Basem Abu Zneid,

Faculty of Electrical and Computer Engineering,

University of Business and Technology CEiT,

Dhaban - North Jeddah, Saudi Arabia.

Email: basem782001@gmail.com

\section{INTRODUCTION}

Microcontroller evolution has attracted considerable interest from researchers in automated applications because of their performance and effectiveness. Microcontroller evolution is used in daily applications in different fields, such as medical applications, automation systems, wireless communications, and control systems. Control systems can be developed using various types of microcomputers, such as PIC, dsPIC microcontroller, and Arduino controller. The primary applications that use PIC16F and Arduino controllers are reviewed in this study. The efficient advantages of these controllers are used to develop the presented applications [1,2].

Engineering software tools such as MATLAB and LabVIEW demonstrate the flexible control of integrated applications [3, 4]. These tools satisfy the need for flexibility, performing measurements, and observing tasks in different fields. Moreover, the increasing demands of the industry to extend the remote controlling and monitoring of various devices are fulfilled. These tools also construct the graphical presentation of measured values based on user requirements and preferences.

\section{MICROCONTROLLER APPLICATIONS}

Smart house applications can be used to perform unlimited tasks, including intelligent parking, which may likely fulfill the aim of managing and protecting cars in residential parking areas. The parking area can be managed and organized with smart technologies and tools based on image recognition technology and the Arduino controller to effectively integrate image-processing technologies [3]. 
Monitoring and measurement can also be implemented to increase efficiency and decrease the cost of specific tasks. Control and design can be achieved with the aid of professional software, such as the National Instrument Laboratory Engineering Workbench (LabVIEW), modular measurement, and control hardware. Equipment can be remotely monitored using virtual instruments. The data can then be saved and analyzed for comparison with traditional tools and provide a safe system for users [4].

Design and implementation of bluetooth video surveillance devices based on microcontrollers are offered in reference [5]. Portable wireless video surveillance devices are widely used due to the bluetooth hardware embedded in their systems. Furthermore, the presented design was intended for video surveillance devices capable of video data transmission between video servers and clients [5]. Various applications have also been developed in different fields, such as syringe pumps, gas leakage warning systems, and smart green houses.

\subsection{Syringe pump}

In this scheme, the development of a controlled injection pump is presented. The planned and manufactured enhanced injection pump is a medical application robot that can be used in health centers. The implementation of this device includes the development of complete mechanical and control systems that use the microcontroller. This device is designed and programmed to activate the pumping of specific amounts of medical solutions as requested by the user. Volume and speed of the fluid are controlled with the assistance of the microcontroller based on user preference.

Figure 1 shows that the proposed system was successfully implemented and functioned satisfactorily. The results revealed good performance of the developed device in terms of accuracy and precision. Furthermore, a flow detection sensor was integrated to monitor the reference value, and the measured value was obtained from the developed device. The proposed system successfully functioned with high accuracy and obtained an average error of approximately less than $1.5 \%$, which could be considered an excellent value compared with those of existing devices. The proposed design has the advantage of supporting the use of commercially available injection syringes. The figure also shows that the system is cost-effective compared with similar devices available in the market.

Finally, the syringe pump module was tested at a fixed speed of $100 \mathrm{ml} / \mathrm{h}$, with volume rate size changes from $2 \mathrm{ml}$ to $10 \mathrm{ml}$. Figure 2 presents the comparison between the reference and measured values. The results show that the proposed model fuctions with satisfactory accuracy at the commonly used speed with reference to similar devices [6].

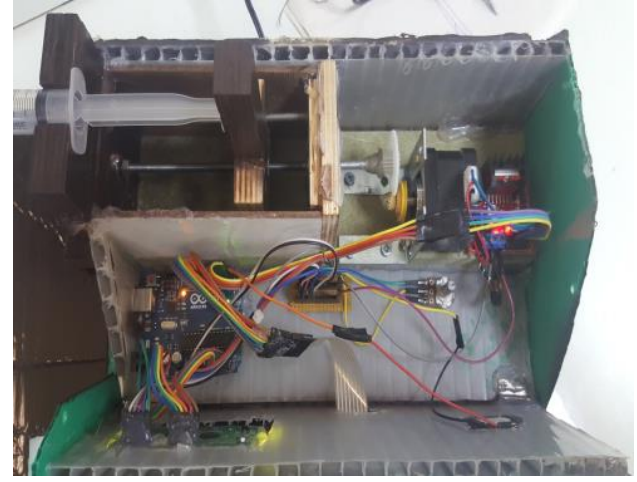

Figure 1. Developed syringe pump system

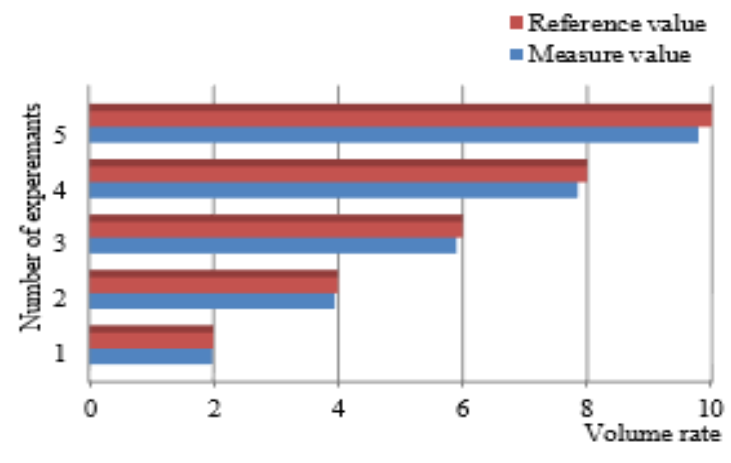

Figure 2. Error at $100 \mathrm{ml} / \mathrm{h}$

\subsection{Gas leakage warning system}

Liquefied petroleum gas (LPG) was invented in 1910 and is now widely used in homes for cooking purposes [7]. Despite the higher cost of LPG compared with new types of fuels, such as fuel wood, LPG is still considered cleaner for home usage owing to its low impact on air pollution [8, 9]. In some cases, gas leaks may cause accidents that can lead to increased financial losses, apart from the human injuries caused by the explosion of gas cylinders. The risk of these explosions has increased in recent years because of external factors, such as corrosion, construction defects, material failures, old valves, worn-out regulators, and lack of awareness in the use of gas cylinders.

To avoid the hazard of a gas leak or fire, a system that warns us of gas leakage is investigated and implemented. Figure 3 shows a general scheme of the proposed gas leakage warning system. The LPG leakage detection system detects leakage by utilizing specific gas sensors with the help of a microcontroller. 
A buzzer is activated to generate a series of warning messages as audio or visual alarms, thereby resulting in the closure of the gas supply valve. A small amount of LPG gas leakage near the sensor is detected and the buzzer activates the audiovisual alarm, causing the gas supply valve to shut down. The consumer and fire station are alerted by the system when the LPG gas leakage reaches a critical level.

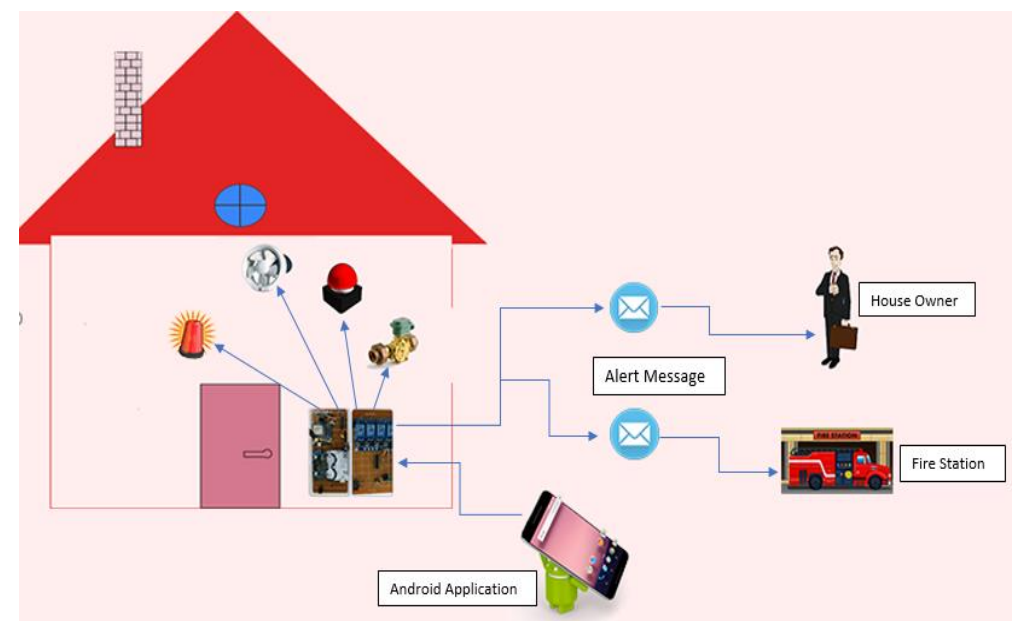

Figure 3. General scheme of a gas leakage warning system

The primary feature of the proposed model is its use of a mobile phone application based on a leak detection gas sensor with the help of a microcontroller, which is needed to proficiently and continuously sense the LPG gas with a rapidly working controller. The sensor, which is used to detect the presence of gas in the air, must be sensitive to propane and butane. The developed scheme senses the leak with the help of the microcontroller, which automatically initiates an instruction for the system to lock the regulator solenoid valve and avoid further leakage from the cylinder. Simultaneously, a buzzer alarm is activated in the room. A message is then sent to the houseowner and the fire station by means of a global system for mobile (GSM) technology. Figure 4 shows the flow of system functionality.

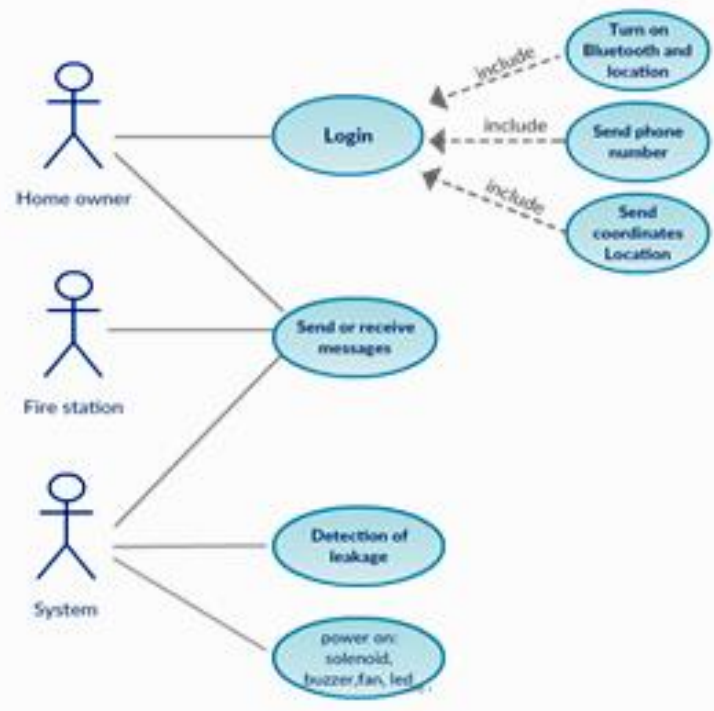

Figure 4. System functionality flowchart 
The designed system contains a wireless transmission system based on Bluetooth, with power supply and detection gas sensor as inputs to the Arduino microcontroller for further processing control. Figure 5 shows the input sensor to resolve the required output of the warning system. The system will then shut down the solenoid valve, activate a buzzer, and transmit alert messages as SMS via GSM.
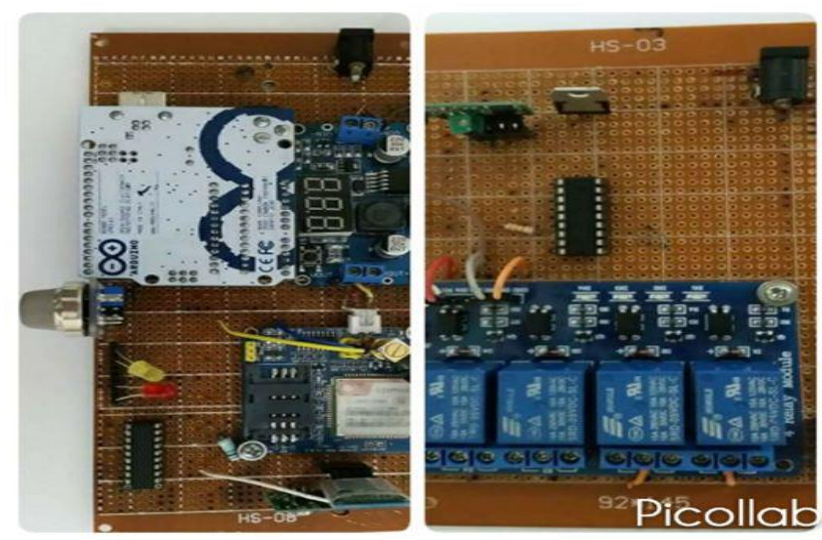

Figure 5. Gas leakage warning system

\subsection{Smart green house}

The proposed remote control system is a model of a Green-House, which uses smart technology to provide suitable plantation environments. The proposed system aims to supply plants and trees with the required nourishment from sunlight and protect them from the harmful effects of the sun. Figure 6 shows the remote control system used with the Arduino microcontroller to adjust humidity and temperature, soil moisture, light sensor, relay nodes for monitoring, and automatic control.

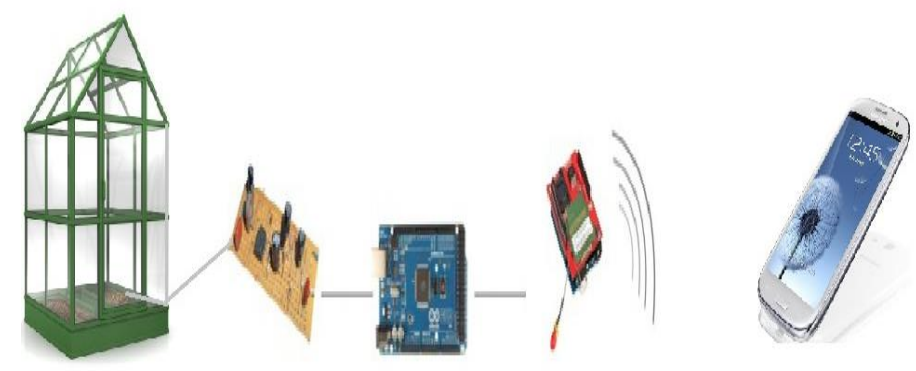

Figure 6. System process

A greenhouse model is developed and proposed to successfully control systems that monitor plant growth by closely analyzing the relationship between indoor environmental information and monitored information, such as temperature and soil humidity. This system also automatically collects information and effectively controls the greenhouse from a remote location through a GSM modem. Figure 7 shows the general flow of the system.

The main advantage of the proposed smart greenhouse is the use of smart technologies, which could make smart greenhouses user friendly at low cost. Consequently, the middle class can afford smart greenhouses and benefit from their convenience and safety. Figure 8 shows the flowchart of how the system works. In the beginning, the system receives analog data measured by the sensor. The system detects the measured quantities and converts the analog data into digital data by using an analog digital converter with the help of the microcontroller. The data are then recorded and transmitted via GSM transmission system. Figure 8 presents the overall flow of the designed programming control code. 


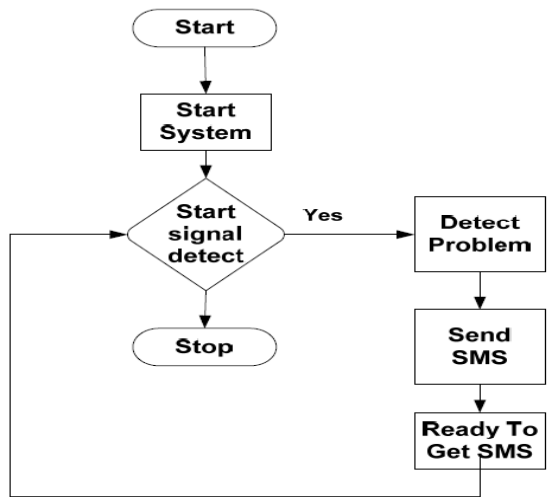

Figure 7. General flow diagram of the system

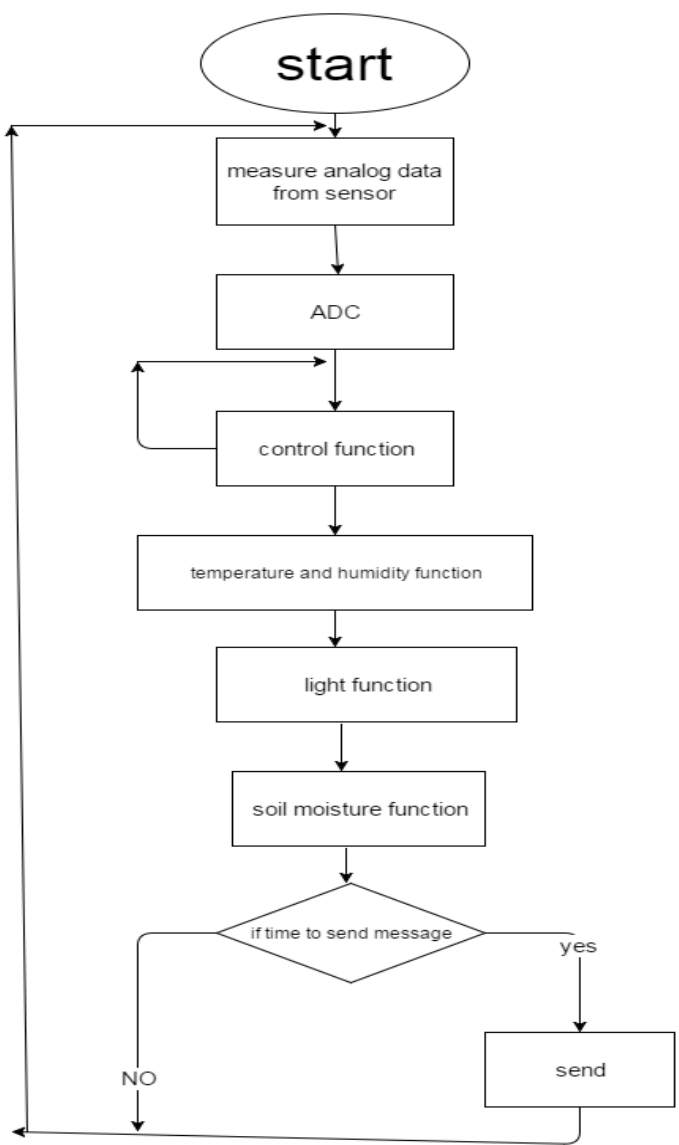

Figure 8. Overall flow of the designed programming control code

This proposed prototype offers a fully automated greenhouse management system. Experiments validate that the system fulfills all the requirements related to greenhouse monitoring. The automatic greenhouse sensor design can assist in increasing plantation productivity. The proposed design provides automatic control over different devices, such as light and motor pump. This design also has the mechanism to alert farmers on parameter changes in the greenhouse to facilitate early precautionary steps. Figure 9 shows the hardware design of the greenhouse monitoring system. The implementation of the proposed design could continuously increase the productivity of cropping, leading to a resolution of the famine problem around the world. In addition, the greenhouse monitoring system can be integrated with GSM to make it better than other systems using different technologies. 


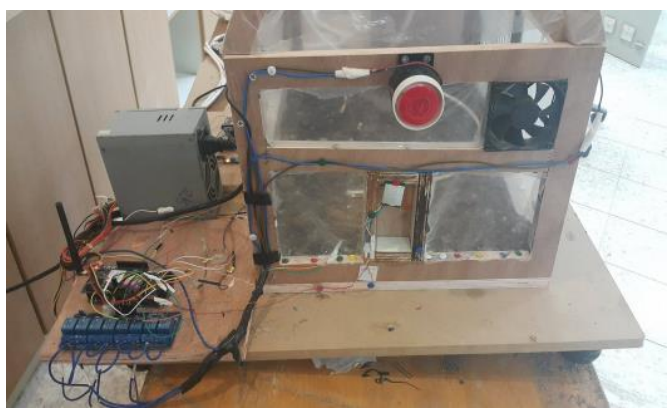

Figure 9. Hardware design of the greenhouse monitoring system

\subsection{Microcontroller and electro-optic technique}

Microcontroller-based systems are simple, low cost, and user friendly. Figure 10 presents a fiberoptic remote monitoring technique designed to measure the $\mathrm{CO}$ concentration in the environment. The proposed design is equipped with an electrochemical sensor array to sense CO contamination and control and monitor air quality. The results obtained from computer analysis and the microcontroller-based model show an increased degree of safety. The remote operation in places under hazardous conditions through the fiber-optic communication network provides the required isolation for such environments. Moreover, monitoring becomes a demand in the detection of toxic gases in the surrounding environment. The detection of these gases is based on different techniques, such as infrared spectrograph technique for $\mathrm{CO}_{2}$ monitoring $[10,11]$.

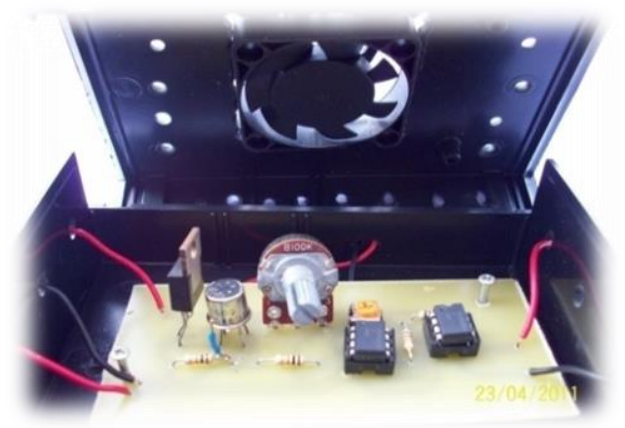

Figure 10. TGS2600 gas sensor measurement circuit

A monitoring system based on the Zigbee technology sensor network for coal mine protection has been developed to collect and record the values of methane concentration. The proposed system is likewise capable of measuring the amount of humidity in underground mines using the Zigbee sensor located inside the coal mine $[12,13]$. The designed model also implements an embedded microcontroller for controlling and monitoring fluid transport in the peristaltic pump. The system electronic hardware mainly consists of STM32F4 development board, stepper motor driver circuit, and current, magnetic, and arterial pressure sensors. Sensor circuits were developed in parallel with the development of embedded software, which mainly consists of different routines. These routines are programmed and designed for monitoring current, pressure, and magnetic sensors, as well as pump speed controller routines. The peristaltic pump system was tested with a mixture of water and glycerin with a viscosity similar to human blood [14].

Furthermore, an automated double-ring infiltrometer (DRI) system has been developed utilizing an Arduino microcontroller. The proposed design includes a Hall effect sensor, peristaltic pump, water level sensor, and constant-level float valve. The designed system is used in single-ring falling head and doublering constant head infiltration measurements. This system operates without the need for a portable computer in the field because real-time data are stored in the attached micro-SD flash memory card. The main requirement for this system is a single reservoir for the inner and outer rings wherein water can be added anytime as needed without affecting the measurements. The proposed design is mounted on a portable and weather-resistant box to test and run the DRI system in the field. The results show that the system is applicable for portable field measurement set-up [15]. 
Figure 11 presents an ultrasound scanner prototype hardware based on the 8051 microcontroller, which is controlled from a host laptop using MATLAB graphical user interface. The microcontroller interfaces with MATLAB software through an intermediate MEX "C" language program to obtain smooth interfacing of the microcontrollers with MATLAB $[12,16]$.

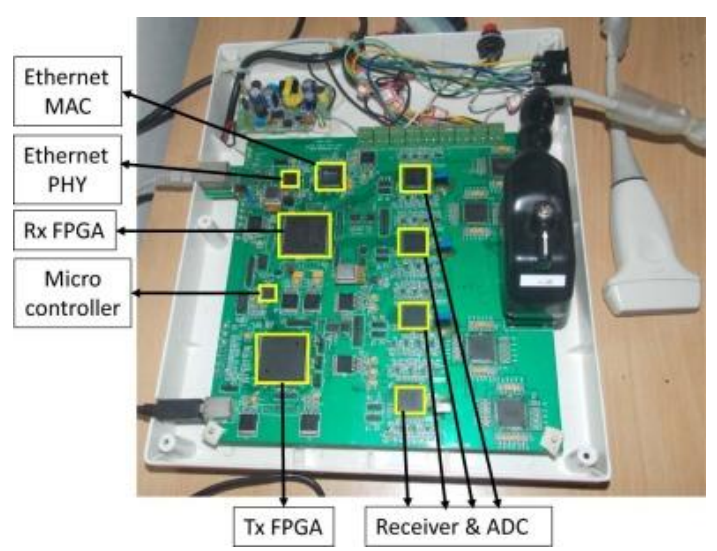

Figure 11. Ultrasound scanner prototype hardware [16]

Another scheme was implemented with the TMDXDOCKH52C1 Concerto Kit and a pair of ultrasound transducers. The presented scheme includes a microcontroller system for transmission, acquisition, and processing of ultrasound signals for nondestructive testing (NDT) of wood samples. The developed system can generate high-amplitude pulses (up to $500 \mathrm{Vpp}$ ) to excite an ultrasonic transducer. The data collected by the system can be used to determine parameters such as velocity, attenuation, and other parameters of ultrasound waves in wood samples for material characterization. The results of system testing show that the developed prototype meets the NDT application requirements [17]. Figure 12 shows the block diagram of the developed system.

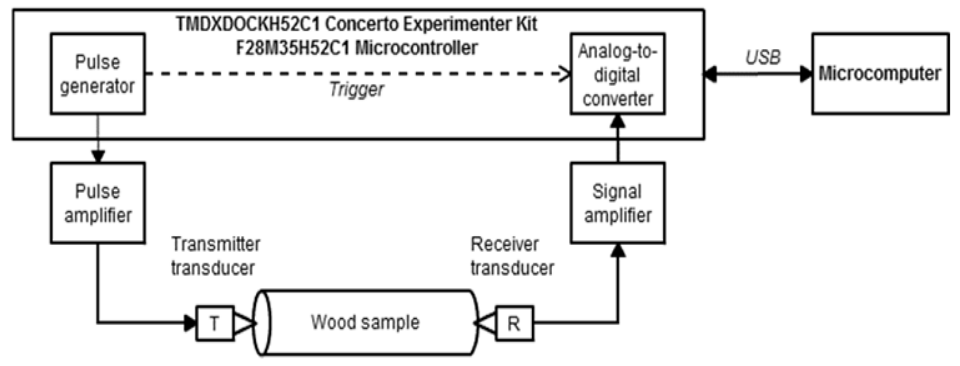

Figure 12. Block diagram of the NDT system [17]

Furthermore, a microcontroller system is designed to control the navigation of a mobile robot and avoid obstacles. The designed scheme functionality is based on ultrasonic sensors that work together with the navigation system. The presented design aimed to operate a mobile robot in a warehouse. The hybrid navigation system combines perception and dead reckoning to obtain satisfactory operation. The transformation of information into a functional form is appropriate for production navigation decisions, which can be considered sensor fusion $[13,18]$.

Figure 13 shows a microcontroller-based interface circuit for inductive sensor measurement. The proposed technique was implemented using a low-cost microcontroller, which appropriately excites RL circuits to measure the discharging time of the voltage across each inductance (i.e., sensing and reference). Then, the measured discharging times are used to estimate sensor inductance. The results show a nonlinearity error lower than $0.5 \%$ at full-scale span when measuring inductances from $1 \mathrm{mH}$ to $10 \mathrm{mH}$ and from $10 \mathrm{mH}$ to $100 \mathrm{mH}$. The circuit only requires an external resistor and a reference inductance in addition to the microcontroller and sensor. Hence, two RL circuits with high-pass filter are formed [14, 19]. 

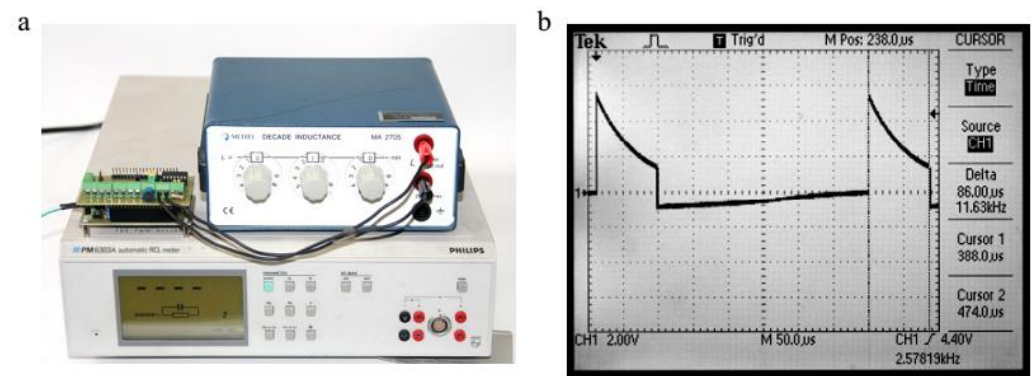

Figure 13. (a) Microcontroller-based interface circuit for an inductive sensor (Lx) and (b) transient response of the voltage at pins 1 and 2 when measuring the RL circuit that includes Lx [14]

A microcontroller-based instrumentation system for air pressure monitoring is also implemented using optical fiber sensors. The developed system consists of a laser source, a beam splitter, two multimode optical fibers, two light-dependent resistance (LDR)-based timer circuits, and an AT89S8252 microcontroller. The beam splitter is used to divide the laser beam into two parts, and then the two beams are launched into two multimode fibers. One of the multimode fibers is used as the sensor fiber, and the other one is utilized as the reference fiber. The reference fiber eliminates the environmental effects disturbing the air-pressure magnitude measurement. The laser beams from the sensor and reference fibers are applied to two identical LDR-based timer circuits. The microcontroller samples the frequencies of the timer circuits using its counters, and then the counter values are processed to identify the measured magnitude of air pressure [20].

A PV emulator that models the electrical characteristic of a photovoltaic (PV) panel is also presented to authenticate the design and testing of PV power systems. The I-V curve of PV is the key characteristic for PV modeling. The proposed method uses piecewise linear approach because of its simplicity in deriving and implementing a low-cost microcontroller. A two-switch buck-boost DC/DC converter is selected as the PV emulator. The experimental results of the developed model of the PV emulator show the effectiveness of the proposed approach [21].

The design and implementation of a chip-type airborne sound warning system is presented. The proposed system mainly consists of a monolithic integrated circuit P87C52X2FN and a voice chip ISD4003-8 $\mathrm{M}$ as the core. The main advantages of the proposed warning system include strong function, high precision, and easy operation [22]. Moreover, a device that controls the E-test strip application is designed. The developed device was implemented based on a PIC16F877A microcontroller. The main function of the developed device is to combine two devices, which are needed for the E-test strip application's stages, into one to reduce the cost. The proposed design was implemented and tested in the laboratory and actual environment. The test results show that the designed system can be used in hospitals [23].

Numerous studies have shown that artificial hypothermia of the brain under the condition of anesthesia with a rectal temperature decreased to $33^{\circ} \mathrm{C}$ produces remarkable prophylactic effects, thereby protecting the brain from anoxia. In the presented study, a microcontroller-based design for the human brain hypothermia system (HBHS) is constructed for cooling and heating the brain. HBHS consists of a thermoelectric hypothermic helmet, a control unit, and a power unit. Helmet temperature is controlled by an 8-bit PIC16F877 microcontroller, which is adjusted between $-5^{\circ} \mathrm{C}$ and $+46^{\circ} \mathrm{C}$ with an accuracy of $+/-0.5^{\circ} \mathrm{C}$ [24]. Moreover, a heating rate controller is obtained using an AT89C55WD microcontroller for the measurement of thermo luminescence (TL) in alkali halides and other related compounds. The proposed device can also measure temperature and the amount of light emitted by the sample for TL studies [25].

An intelligent distributed controller is designed for freshwater aquaculture plant. The proposed design is based on an ATmega8535 microcontroller platform that enables the control of various environmental parameters under optimum conditions. The presented design is a compact, fast, flexible, distributed, and cost-effective solution for the control of required parameters, such as temperature, lighting, dissolved oxygen, and $\mathrm{pH}$ values [26]. Another research presents the design and development of a resonant sensor for low range (0-10 grams) mass measurement. The use of the microcontroller in the proposed sensor has the advantage of easily extending the measured quantities to any range [27].

To measure the concentration of sodium in blood serum, a low-cost microcontroller-based sodium analyzer is developed. The proposed design is based on an ATmega8535 microcontroller and its associated peripherals. The sodium ion selective electrode is used as a sensor for the measurement. A platform is developed in " $\mathrm{C}$ " language to measure, analyze, and display the collected data. The results of sodium concentration measurement from the collected samples validate the effective performance of the designed instrument with the aid of linear regression analysis [28]. 
Similarly, a microcontroller-based repetitive transcranial magnetic stimulator (TMS) was developed and tested at low voltage levels. Design parameters of the TMS were determined and three PIC microcontrollers were used to control these parameters in the developed system. The frequency of stimulation pulses can be set to a predetermined value via the microcontroller, wherein one of the outputs is pulse width modulation (PWM). The other advantage of the designed system is the remote control of stimulation pulse, which uses ASK modulation technique. A performance test of the system was conducted and stimulation pulse was analyzed [29].

The design and test of the digital temperature control system is presented by reference [30]. The developed system uses an algorithm that implements a $100 \%$ duty cycle for temperature errors of $2^{\circ} \mathrm{C}$ or higher and a $25 \%$ duty cycle for temperature errors of $1{ }^{\circ} \mathrm{C}$ for the controller. The temperature rate measurements were constant for positive and negative temperature gradients. The system also enabled the incubator to reach the steady-state temperature in less than $70 \mathrm{~s}$ [30].

A microcontroller-based system for dielectric constant measurement in liquids was designed and developed. The system measures the change in frequency of an XR-2206 function generator using an Atmel's AT89C51 microcontroller once the liquid forms the dielectric medium of the dielectric cell. The proposed system covers a wide range of dielectric constants for various liquids at various concentrations and at different temperatures [31]. In addition, a polarographic-type oxygen gas sensor and a gas concentration controller circuit are designed for medical applications. A case example, such as incubator environments, dries the air of the incubator environment during the control processes, and the oxygen gas flow is humidified by the designed ultrasonic nebulizer. The signal conditioning circuit obtains the required voltage levels and control processes using a high-speed PIC microcontroller [32].

A BLDC motor drive system is an example of an industrial application of microcontroller-based systems. A microcontroller-based technique is proposed to minimize the torque ripples from current commutation to improve the performance of the BLDC drive system. This study presents a new switching technique that has been successfully implemented using a mid-range PIC microcontroller, which is commercially available at an affordable price. The PIC18F is used to generate the required PWM signals and control the power switches. Compared with conventional technique, the results of the proposed system validate its effectiveness with smoother output torque and current with 50\% reduction of torque ripples [33]. These motors are now widespread due to the advancement of digital controllers in high-demand applications, such as electric vehicles [34]. Research on biological signal measurement systems using a smartphone is presented. The proposed system mainly consists of an instrumentation amplifier, filter, and AC/DC converter. The useful electrical signal with the aid of an algorithm parallelizes the process and can execute the output of earphone analog signal and microphone terminal at the same time. The experimental results demonstrate that the presented scheme operates properly [35].

Finally, this review paper presents the different developed low-cost and smart techniques for safety and remote monitoring applications. Such applications are integrated with smartphone tools for end users' convenience. The developed techniques may be implemented in the industry or the market because of their user-friendly platform and affordable prices. The use of microcontrollers in different home and industrial applications helps to implement up-to-date technology, such as easy integration with Internet of Things (IoT). IoT technology has the advantages of connecting with everything in the world and being green or environmentally friendly due to its low power consumption [36].

\section{CONCLUSION}

An overview is conducted on the evolution of a microcontroller with low-cost and user-friendly applications for safety and health monitoring, and smarthouse applications. In addition, these microcontroller-based smart devices can be used in industrial applications, such as the BLDC drive systems. These systems can be obtained using microcontroller devices with wireless communication units. The applications proposed in this work aimed to review the significance of new technology and mobile phone applications to implement different developed techniques. Accordingly, an automated building, which is integrated with today's smart technologies, can help realize a convenient and secure daily life. The proposed implemented applications show good performance and rapid response. Long-life devices can likewise be used anywhere to fulfill the required job.

\section{ACKNOWLEDGEMENTS}

The authors would like to thank Palestine Technical University-Kadoorie (PTUK) and the Palestinian Ministry of Education and Higher Education for funding this research. In addition, the authors thank the technicians and undergraduate students who provided technical assistance and lab work support. 


\section{REFERENCES}

[1] M. Rehman, M. Abdul Mujeebu, T. B. Kheng, and B. A. J. A. Abu Izneid, "A Microprocessor-Based Novel Instrument for Temperature and Thermal Conductivity Measurements," Experimental Techniques, vol. 36, pp. 62-70, 2012.

[2] B. A. Izneid, I. Sukar, M. Ali, and M. Souiyah, "Development of wireless Bluetooth heart rate remote monitoring system," in IET International Conference on Wireless Communications and Applications (ICWCA 2012), pp. 1-4, 2012.

[3] A. Abu Sneineh and W. A. Salah, "Palestine Automotive License Identity Recognition for Intelligent Parking System," Journal of Engineering Science and Technology, vol. 12(5), 2017.

[4] W. A. Salah, A. B. Musa, B. A. Zneid, A. A. Sneineh, and M. S. Jadin, "Implementation of Virtual Instruments as a Power Quality Analysis Tool," Journal of Low Power Electronics, vol. 12, pp. 83-90, 2016.

[5] Y. Yu, X. Duan, S. Wang, and B. Jiao, "The design and implementation of bluetooth video surveillance devices," in 2010 3rd International Congress on Image and Signal Processing, pp. 495-498, 2010.

[6] W. A. Salah and A. A. Sneineh, "Exploring the Knowledge and Attitude of Engineering Students in the Imitation of Theoretical Knowledge," Modern Applied Science, vol. 11, pp. 74-78, 2017.

[7] M. Marchionna, R. Patrini, D. Sanfilippo, and G. Migliavacca, "Fundamental investigations on di-methyl ether (DME) as LPG substitute or make-up for domestic uses," Fuel Processing Technology, vol. 89(12), pp. 1255-1261, 2008.

[8] A. N. Anozie, A. R. Bakare, J. A. Sonibare, and T. O. Oyebisi, "Evaluation of cooking energy cost, efficiency, impact on air pollution and policy in Nigeria," Energy, vol. 32(7), pp. 1283-1290, 2007.

[9] S. D. Pohekar, D. Kumar, and M. Ramachandran, "Dissemination of cooking energy alternatives in India a review," Renewable and Sustainable Energy Reviews, vol. 9(8), pp. 379-393, 2005.

[10] B. A. Zneid, M. Al-zidi, and T. Al-kharazi, "Non-invasive blood pressure remote monitoring instrument based microcontroller," in 2014 IEEE Region 10 Symposium, pp. 248-253, 2014

[11] Basem Abu Izneid, Ahmed Abdulrahman, Tareq Al-kharazi, "Development of remote monitoring (CO) measurement instrument based microcontroller and electro-optic technique," 2013 IEEE International RF and Microwave Conference (RFM), pp. 433-436, 2014.

[12] K. Mollgaard, "Acoustic gas measurement", Biomedical Instrumentation and Technology," pp.495-7, 1989.

[13] L.Yu, A.Li, Z.Sun, and H, Li "Design of Monitoring System for Coal Mine Safety Based on Wireless Sensor Network," in IEEE/ASME International Conference on Mechtronic and Embedded Systems and Applications (MESA2008), pp. 409-414, 2008.

[14] Pratondo Busonoa, Andi Iswahyudib, M. Akbar Aulia Rahmanb, Ario Fitriantoa, "Design of Embedded Microcontroller for Controlling and Monitoring Blood Pump," Procedia Computer Science, vol. 72, pp. 217-224, 2015.

[15] M. Fatehniaa, S. Paranb, S. Kishc K., Tawfiqa, "Automating double ring infiltrometer with an Arduino microcontroller," Geoderma, vol. 262, pp. 133-13915, January 2016.

[16] Raj, Jean Rossario, S. M. K. Rahman, and Sneh Anand. "Microcontroller USB interfacing with MATLAB GUI for low cost medical ultrasound scanners," Engineering Science and Technology, an International Journal, vol. 19(2), pp. 964-969, 2016.

[17] Fabiano C. DomingosaJoaquim M. Maiaa Ozana M. A. Maiab Fábio K. Schneidera, "Microcontroller based Control System for Ultrasound NDT in Wood," Physics Procedia, vol. 70, pp. 428-432, 2015.

[18] Aziza M .Zaki, Osama Arafa,Sanaa I. Amer, "Microcontroller-based mobile robot positioning and obstacle avoidance," Journal of Electrical Systems and Information Technology, vol. 1(1), pp. 58-71, May 2014.

[19] Kokolanski, Zivko, José Jordana Barnils, Manuel Gasulla Forner, Vladimir Dimcev, and Ferran Reverter Cubarsí. "Microcontroller-based interface circuit for inductive sensors," vol. 87, pp. 1251-1254, 2014.

[20] D. Hazarika, D. S. Pegu1, "Micro-controller based air pressure monitoring instrumentation system using optical fibers as sensor," Optical Fiber Technology, vol. 19(2), pp. 83-87, March 2013.

[21] Dylan D. C. Lu, Quang Ngoc Nguyen, "A photovoltaic panel emulator using a buck-boost DC/DC converter and a low cost micro-controller," Solar Energy, vol. 86(5), pp. 1477-1484, May 2012.

[22] Shiyanbina GUO Jianb SHI Yanlic, "Design of the Sound-Warning System based on the Micro-Controller," Physics Procedia, vol. 25, pp. 1301-1306, 2012.

[23] Bahar Cilik,Nihal Fatma, İnan Güler, "Design and Realization of a Microcontroller Based E-Test Strip Application Device," Instrumentation Science \& Technology, vol. 37(6), pp. 676-682, 2009.

[24] Kapidere M., Ahiska R., Güler I., "A new microcontroller-based human brain hypothermia system," Journal of Medical Systems archive, vol. 29(5), pp. 501-512, October 2005.

[25] A. Rajendran, P. Neelamegam, "Automated Heating Rate Controller for Thermo luminescence Measurements Using a Microcontroller," Instrumentation Science \& Technology, vol. 32(4), pp. 379-386, 2004.

[26] P. Neelamegam,S. Kumaravel \& R. Raghunathan, "Microcontroller Based Distributed Monitoring System for Fresh Water Fish Aquaculture," Instrumentation Science \& Technology, vol. 36(5), pp. 515-524, 2008.

[27] G. Uma, M. Umapathy, A. M. Josephine, S. Aishwarya \& K. Suresh, "Design of Microcontroller Based Resonant Sensor with Piezoelectric Excitation and Detection," Instrumentation Science and Technology, vol. 36(4), pp. 367-374, 2008.

[28] P. Neelamegam, K. Murugananthan, R. Raghunathan \& A. Jamaludeen, "ATmega8535 Microcontroller Based Blood Sodium Analyzer Using Ise Direct Potentiometry," Instrumentation Science \& Technology, Volume 38 (1), pp. 63-71, 2009. 
[29] M. Burunkaya \& I. Guler, "Design and Construction of a Microcontroller Based Transcranial Magnetic Stimulator Instrumentation," Science \& Technology, vol. 36(1), pp. 32-42, 2007.

[30] Kaitano Dzinavatonga, "Interface Of A Digital Temperature Microcontroller With An Analog Incubator," Instrumentation Science \& Technology, vol. 42(1), pp. 48-45, 2014.

[31] Ch. V. V. Ramana \& K. Malakondaiah, "Microcontroller Based System for the Measurement of Dielectric Constant in Liquids," Instrumentation Science \& Technology, vol. 35, pp. 599-608, 2007.

[32] M. Burunkaya \& Prof. I., "Guler A Microcontroller Based, Low Cost Oxygen Gas Controller for Medical Purposes," Instrumentation Science \& Technology, vol. 34(5), pp. 509-527, 2006.

[33] W. A. Salah, Ishak, D., Zneid, B. A., Abu_Al_Aish, A., Jadin, M. S., \& Sneineh, A. A., "Implementation of PWM control strategy for torque ripples reduction in brushless DC motors," Electrical Engineering, vol. 97(3), pp. 239-250, 2015.

[34] Wael A. Salah, Mahmoud A. M. Albreem, Basim Alsayid, Basem Abu Zneid, Mutasem Alkhasawneh, Anwar Al-Mofleh, Anees Abu Sneineh, Amir Abu Al-Aish, "Electric vehicle technology impacts on energy," International Journal of Power Electronics and Drive Systems (IJPEDS), vol. 10(1), pp. 1-9, 2019.

[35] Ryoichi Miyauchi, Koichi Tanno, Hiroki Tamura, "Simple Measurement System for Biological Signal Using a Smartphone," International Journal of Electrical and Computer Engineering (IJECE) ,vol. 8(6), pp. 4157-4163, December 2018.

[36] Mahmoud A. M. Albreem; Ayman A. El-Saleh; Muzamir Isa; Wael Salah; M. Jusoh; M.M Azizan; A Ali, "Green Internet of Things (IoT): An overview," in the 2017 IEEE 4th International Conference on Smart Instrumentation, Measurement and Application (ICSIMA), pp. 1-6, Putrajaya, 2017.

\section{BIOGRAPHIES OF AUTHORS}

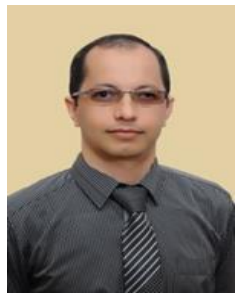

Wael A. Salah was born in Hebron, Palestine in 1978. He received his Bachelor degree in Electrical and Computer Engineering from Palestine Polytechnic University in 2001. He obtained his MS and $\mathrm{PhD}$ degrees in Electrical and Electronic Engineering from Universiti Sains Malaysia in 2007 and 2012, respectively. From October 2012 until July 2014, Dr. Salah was connected with the Faculty of Engineering of Multimedia University. Currently, he is an associate professor at Palestine Technical University in Khadoori. His research interests include power electronics and electric drives, energy management, energy efficiency, power control and management, and renewable energy systems. Dr. Salah has published more than 40 papers in international journals and conferences and is a recognized board member for several international journals and conference proceedings. $\mathrm{He}$ is a member of the Jordanian Engineers Association and Arab Engineers Association and is a Senior Member of the Institute of Electrical and Electronics Engineers in the US.

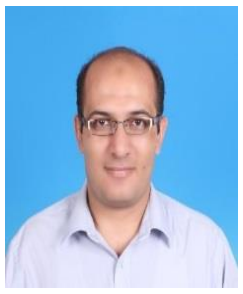

Basem Abu Zneid received his PhD degree in Electrical and Electronic Engineering from Universiti Sains Malaysia in 2010. He is an assistant professor at the University of Business and Technology . CEiT, Saudi Arabia. 